BEST EVIDENCE TOPIC REPORTS

\title{
Towards evidence-based emergency medicine: best BETs from the Manchester Royal Infirmary
}

\author{
Edited by Simon D Carley
}

Emerg Med J 2006;23:872-877. doi: 10.1136/emj.2006.042143

Best evidence topic reports (BETs) summarise the evidence pertaining to particular clinical questions. They are not systematic reviews, but contain the best (highest level) evidence that can be practically obtained by busy practising clinicians. The search strategies used to find the best evidence are reported in detail to allow clinicians to update searches whenever necessary. Each BET is based on a clinical scenario and ends with a clinical bottomline that indicates, in the light of the evidence found, what the reporting clinician would do if faced with the same scenario again.

The BETs published below were first reported at the Critical Appraisal Journal Club at the Manchester Royal Infirmary ${ }^{1}$ or placed on the bestbets website. Each BET has been constructed in the four stages that have been described elsewhere ${ }^{2}$. The BETs shown here, together with those published previously and those currently under construction, can be seen at http://www.bestbets.org3. Four BETs are included in this issue of the journal.

- Does normal-shaped pupil exclude the diagnosis of iritis

- Use of lidocaine in the GI cocktail for the treatment of dyspepsia

- Dose $>50 \mathrm{mg} / \mathrm{kg}$ : anticipate fluctuation in mental status with intermittent agitation followed by coma, which would require intubation and ventilation within $12 \mathrm{~h}$

- Diagnosis of drug overdose by rapid reversal with naloxone

- Pregnancy-associated plasma protein: a novel cardiac marker with promise

Where a symbol is attached, it means that a critical appraisal of that paper has been posted on the bestbets website.

Does a normal-shaped pupil exclude the diagnosis of iritis? Use of lidocaine in the gastriointestinal cocktail for the treatment of dyspepsia.

Diagnosis of drug overdose by rapid reversal with naloxone. PAPP-A: a novel cardiac marker with promise.

1 Carley SD, Mackway-Jones K, Jones A, et al. Moving towards evidence based emergency medicine: use of a structured critical appraisal journal club. J Accid Emerg Med 1998;15:220-2.

2 Mackway-Jones K, Carley SD, Morton RJ, et al. The best evidence topic report: a modified CAT for summarising the available evidence in emergency medicine. J Accid Emerg Med 1998;15:222-6.

3 Mackway-Jones K, Carley SD. bestbets.org: Odds on favourite for evidence in emergency medicine reaches the worldwide web. J Accid Emerg Med 2000;17:235-6.

\section{Does a normal-shaped pupil exclude the diagnosis of iritis}

\section{Report by Thomas Hunsley, Medical Student, Manchester Medical School, Manchester, UK Checked by Caroline Lee, SpR Emergency Medicine Midland rotation, UK} doi: 10.1136/emj.2006.042150

A short-cut review was carried out to establish whether an abnormal pupil was a highly sensitive marker for acute iritis.
A total of 174 citations were reviewed, of which one answered the three-part question. The clinical bottomline is that patients who present with an acute iritis (anterior uveitis) often have no change in pupil size or shape-that is, their pupil is normal compared with their other eye.

\section{Three-part question}

In [patients with suspected iritis] does [the presence of a normal pupil] exclude [the diagnosis of iritis]?

\section{Clinical scenario}

A patient presents to the emergency department with a unilateral, red, painful eye. Visual acuity is reduced in the affected eye. The pupil is normal and the cornea is clear. There is some ciliary flush around the iris. You can't decide whether the diagnosis of iritis is correct or not. The other features of iritis (ie, unilateral, red, painful and decreased visual acuity) are present, but the pupil remains of normal size and shape. Is this a common presentation?

\section{Search strategy}

Ovid MEDLINE(R) 1966 to August Week 5 2006, Embase 1980 to 2006 Week 25, CINAHL 1982 to June Week 32006 \{[Iritis.mp. or exp Iritis or Anterior uveitis.mp. or exp Uveitis, Anterior or exp Uveitis or exp iridocyclitis PR cyc;it is.mp] and [Pupil.mp. or exp Pupil or exp Adhesions or Adhesions.mp. or posterior synechiae.mp. or Iris.mp. or exp Iris or irregular pupil.mp.] and [exp Diagnosis/or Diagnosis.mp.]\} LIMIT to [humans and english language]

Cochrane Iritis and (Pupil Size or Irregular Pupil)

\section{Search outcome}

Medline 313 papers were found in Medline, of which only one was relevant to the question. This is listed in the table below. No further papers were gained from searching Embase, CINHAL or the Cochrane databases.

\section{Comment(s)}

From the review of the literature, only one paper was found to look into the examination of the eye in acute anterior uveitis. Seven of the eyes presented with a change in pupil size compared with the other normal eye. In all cases, the pupil was miosed (small). All the other 18 eyes presenting with anterior uveitis had normal pupils. The paper states that the formation of posterior synechiae (adhesions between the iris and the lens) is the cause of the miosed pupil. Posterior synechiae is considered to be a complication of prolonged inflammation in the eye. Hence, in the early stages of a first presentation of acute anterior uveitis, there may be no adhesions and therefore the pupil will be normal. An irregular pupil may be seen only after posterior synechiae have formed owing to chronic inflammation, usually as the pupil is artificially dilated.

In another study on iridocyclitis in patients with juvenile rheumatoid arthritis, $61 \%$ of their patients enroled had posterior synechiae.[1] 